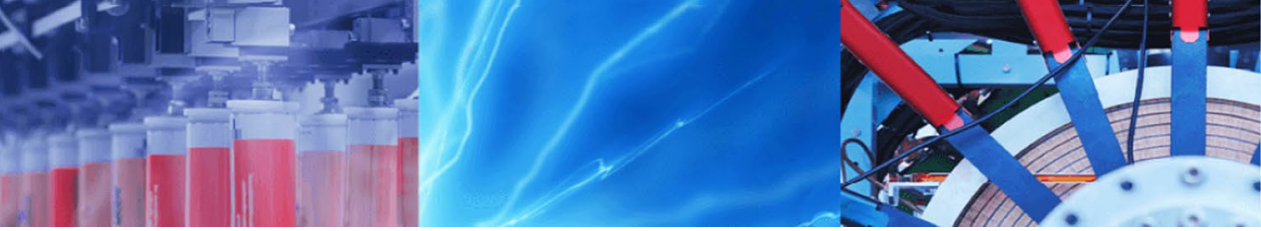

Research Article

\title{
Advancement of weather-related crash prediction model using nonparametric machine learning algorithms
}

\author{
Amit Ranjan Mondal ${ }^{1} \cdot$ Md Abul Ehsan Bhuiyan $^{2}$ (D) Feifei Yang $^{1}$
}

Received: 8 November 2019 / Accepted: 9 July 2020 / Published online: 14 July 2020

(c) Springer Nature Switzerland AG 2020

\begin{abstract}
This paper evaluates the machine learning-based weather-related crash prediction model in Connecticut. Crash severity prediction has always been the principal focus of safety professionals and emergency responders for appropriate policy making and resource management. Over the years, different statistical methodologies (e.g., random forest, support vector machine) have been explored in various research efforts to develop efficient crash severity prediction models. As technology is advancing and computing has started becoming more efficient, machine learning-based models for crash severity prediction are being brought into light for more accurate data-driven prediction. However, some machine learning methodologies provide increased efficiency and better performance compared to others from the same genre. To explore different machine learning methodologies for crash severity prediction, this study considered two machine learning applications_-random forest (RF) and bayesian additive regression trees (BART) for performance comparison. RF model produced higher prediction probabilities for determining crash severity compare to BART. The results were evaluated using various prediction probability analyses obtained from these two machine learning models and showed the model capabilities to generate prediction consistent with the observed data. We found the performance of the RF model to be highly promising with a higher skill score (0.73) than BART (0.61). Overall, our findings demonstrate the robust performances of the RF algorithm in predicting weather-related crashes. The analysis of this study confirms that stakeholders can use weather-related RF model with confidence to obtain a better prediction of crash severity that enables them to facilitate appropriate emergency responses and support essential preemptive measures.
\end{abstract}

Keywords Machine learning $\cdot$ Crash severity $\cdot$ Traffic $\cdot$ Random forest $\cdot$ Bayesian additive regression trees

\section{Introduction}

Adverse weather is the leading cause of crash occurrence which is a major challenge for stakeholder groups [22, 39]. A weather-related accident occurs in any severe condition such as snow, rain, and winds or on slick pavement $[23,39]$. Specifically, $73 \%$ of all weather-related accidents occur when the roads are wet due to rainstorms [39]. The incident of weather-related crashes can be assessed by evaluating adverse weather conditions [39]. Over the past few decades, crash severity prediction due to inclement weather conditions has been the focus of extensive studies conducted by various stakeholder agencies and professional bodies to enhance the safe environment for road users and to minimize the economic cost in responding to such emergencies. It also benefits the agencies and associated organizations to effectively plan for their crash severity mitigation policies and emergency response strategies. Past studies showed that a wide range of crash factors such as pedestrian, driver, roadway, land-use characteristics, along with environmental, behavioral and spatiotemporal characteristics of the roadway and associated

Md Abul Ehsan Bhuiyan, md.bhuiyan@uconn.edu | 'Department of Civil and Environmental Engineering, University of Connecticut, Storrs, CT, USA. ${ }^{2}$ Department of Natural Resources and the Environment, University of Connecticut, Storrs, CT 06269, USA. 
vehicles are considered in crash severity prediction models $[5,18]$. In most cases, this information is reported by the police officers and released in the form of a crash dataset by the governing agencies. The dataset contains valuable information that helps to understand the relationship of various contributing factors in determining the level of injury severity associated with those incidents. The weather-related crash prediction models identify the impacts of those contributing factors and enable one to predict the crash severity based on those contributing factors for better decision making and effective strategy implementation.

Transportation safety professionals and researchers have been implementing statistical approaches as a popular modeling framework for crash analysis and severity prediction. There have been several research attempts to predict the severity of crashes using binary discrete outcome models such as Logit or Probit [19, 29, 40]. For multiple discrete outcomes, multinomial discrete modeling approaches have been used to predict the different level of crash severity [38,42]. Nested Logit approaches have been found in several studies that incorporate the correlation of unobserved factors impacting the level of crash severity [41, 43]. However, the statistical approaches are based on pre-defined modeling techniques and assume a pre-determined nature of the relationship between the prediction and the explanatory variables. As a result, these modeling approaches may lead to significant inaccuracy in severity predictions when the usual assumptions are disproved by the underlying pattern of relationship between the dependent variable and independent variables $[1,13]$.

Machine learning $(\mathrm{ML})$ approaches have been considered a feasible alternative that deals with the traditional shortcomings of statistical approaches and can leverage the underlying relationship patterns to extract knowledgebased information. In recent years, there have been many evolving pieces of research that implemented Artificial Neural Networks in crash severity analysis and prediction $[3,4,17]$. Decision tree algorithms have also been common approaches found in several kinds of literature $[1,14$, 35]. A group of researchers has also implemented Bayesian Networks (BN) approaches for crash severity modeling and reported that it is possible to reduce the set of explanatory variables without degrading the performance in $\mathrm{BN}$-based approaches [36]. However, no major machine learningbased weather-related crash prediction model has been developed to investigate crash severity in the places where adverse weather conditions (such as severe snowstorms, foggy weather, etc.) play a restrictive role in safe driving. Therefore, this study recognizes the necessity to bridge the gap by investigating the severity of crashes in Connecticut, a state in the US where winter weather brings several factors in the roadway that adversely impact the ability of drivers to drive and interact in a safe and knowledgeable manner.

When it comes to implementing the ML approaches, there have been significant efforts in studying the crash severity using the popular machine learning algorithms. $\mathrm{K}$-means clustering $(\mathrm{KC})$ has been used in several studies for crash severity analysis and prediction $[2,37,45]$. Latent class clustering (LCC) is another popular data clustering technique that has been applied in some research studies to address heterogeneity in crash data [19, 21, 32]. A study has found that it cannot be generalized whether $\mathrm{KC}$ or LLC is a better performer in crash severity prediction due to its performance variability based on the crash dataset under consideration [34]. For classification and regression analysis, support vector machines (SVM) and random forests (RF) are two common approaches used in machine learning. SVM has been reported as a better crash severity predicting method compared to ordered probit models $[14,33]$. RF has been implemented in a few studies for crash severity prediction and has been asserted as a functional algorithm that provides satisfactory results $[20,24]$. RF is a powerful and well established non-parametric ensemble classification and regression technique [7] which offers specific features that make it attractive and one of the most accurate learning algorithms for different applications. Moreover, random forest runs efficiently on large datasets and can capture non-linear association patterns between predictors and response. To compare the RF model, we introduced another ensemble-based machine learning technique, Bayesian Additive Regression Trees (BART) [12] which is immensely popular in weather impact modeling, natural hazard, remote sensing, and relevant application $[8,15,27,44]$. Since few nonparametric techniques have so far been developed, we materialized two high-performance nonparametric machine learning techniques (RF and BART) to enhance the weather-related crash prediction model with binary outcomes. Moreover, these two techniques (RF and BART) have been successfully adapted to big data applications and may be suitable to overcome the limitations of the parametric techniques. This is the basic idea of choosing nonparametric ensemble-based ML techniques over traditional parametric methods. Furthermore, this study addresses the need to identify the predictors that provide useful information in predicting crash severity.

Bhuiyan et al. [9-11] developed a machine learningbased multisource data blending technique to evaluate the impact of weather variables on machine learning error modeling. In this study, the technique was extended for the evaluation of the predictive model for crash severity in Connecticut. The primary objectives of this research are to (1) identify the set of important explanatory variables in the crash dataset that can be used to predict severe and 
non-severe crashes, and (2) develop two machine learning-based (RF and BART) models to predict the binary levels of crash severity. The remaining part of this paper is structured as follows: Sect. 2 includes the description of the crash dataset and the case study considered in this research. Section 3 describes the prediction models, validation methodology, and performance evaluation metrics. In Sect. 4, the evaluation results and sensitivity analysis of the case study are discussed briefly. Conclusions and recommendations are discussed in Sect. 5.

\section{Study area and dataset}

A case study was conducted to demonstrate the applicability and feasibility of the proposed crash severity prediction models. The crash information was extracted from the Connecticut Crash Data Repository (CTCDR) for 4 years (2015-2018). The dataset contains information about 435,140 crashes that occurred in the state of Connecticut and were reported by the state and local Police officers. Each crash has been reported with the corresponding weather conditions along with the identification features, environmental conditions, site characteristics, vehicle information, and spatiotemporal attributes. According to the KABCO Injury Scale by Federal Highway Administration (FHWA) of the USA, $75.75 \%$ crashes in the dataset were categorized as " $\mathrm{O}$ " meaning that there were no signs of physical injury and only property damages were reported. The most severe injury category- " $K$ " constitutes only $0.25 \%$ of the total crashes. Though the percentage seems very trivial, the numerical entity somehow appears to be frightening. Almost 1082 casualties were reported over the 4 years in the state of Connecticut leading to an alarming casualty rate of 22.5 casualties per month. The study considers several types of variables that are available in CTCDR datasets to predict crash severity such as temporal variables (month, day of the week, hour of the day), roadway characteristics (route class, type of intersection), environmental (weather condition, light condition, school bus related, work zone related), and crash related (manner of crash/collision impact, most severe injury).

For this study, the crash severity is classified into two categories using the afore-mentioned $\mathrm{KABCO}$ scale: severe injury ( $K$ and $A$ ) and non-severe injury $(B, C$, and $O)$. The crash severity was considered as the response variable in this study, whereas 11 variables (manner of crash, weather condition, route class, hour of the day, type of intersection, light condition, road surface condition, month, work zone related, day of the work, school bus related) were used as the explanatory variable in the models. The spatial distribution of these two categories shows that the locations of severe crashes are clustered in urban clusters in the state as illustrated in Fig. 1. However, non-severe crashes were reported almost uniformly in terms of their spatial distribution.

Most crashes were reported in local and state routes followed by interstate highways and US routes. In terms of the time of day, most non-severe crashes occurred during the afternoon peak period (4 PM-5 PM) of the day followed by the morning peak period (7 AM-8 AM) as shown in Fig. 2. However, the trend for severe crashes shows a different pattern with increasing occurrence during the later part of the day. An interesting finding in this illustration is that there are fewer crashes in Connecticut in 2018.

\section{Methodology}

In this analysis, we created a weather-related crash prediction model using two machine learning (ML) algorithms$\mathrm{RF}$ and BART. The workflow for the framework used in this study is illustrated in Fig. 3.

\subsection{Random forest (RF)}

Random forest (RF) is one of the machine learning applications considered in this study for crash severity prediction. RF is an ensemble-based learning method that can be used for classification or regression. The basic concept of RF technique is to construct multiple decision trees during the learning phase, and then combine the outputs from those decision trees to find out the class in case of classification or mean value of each decision tree in case of regression [25, 26]. To learn the highly unusual patterns of the training dataset, traditional decision trees can go deep resulting in very low bias and considerably high variance. The multiple decision trees in RF models are trained using different chunks of the same training dataset to eliminate this overfitting tendency by lowering the variance [28]. The very first algorithm for $\mathrm{RF}$ is based on the random subspace method $[25,26]$. The bootstrap aggregating approach is an extension of the random subspace algorithm that constructs multiple decision trees in RF models with regulated variance $[6,7]$. Given the set of predictors, $X=X_{1}, X_{2}, \ldots X_{n}$ with respective response variables $Y=Y_{1}, Y_{2}, \ldots Y_{n}$, the bootstrap bagging technique is used to repeatedly select a random training sample with replacement and generates tree $\left(f_{b}\right)$ for each repetition. Here, $Y$ represents the crash severity as a response variable, whereas $X$ indicates 11 explanatory variables (manner of crash, weather condition, route class, hour of the day, type of intersection, light condition, road surface condition, month, work zone related, day of the work, school bus related) for the model in this study. When the training is complete after $B$ repetitions, the prediction can be made on an unknown sample by calculating the average of predictions from all 

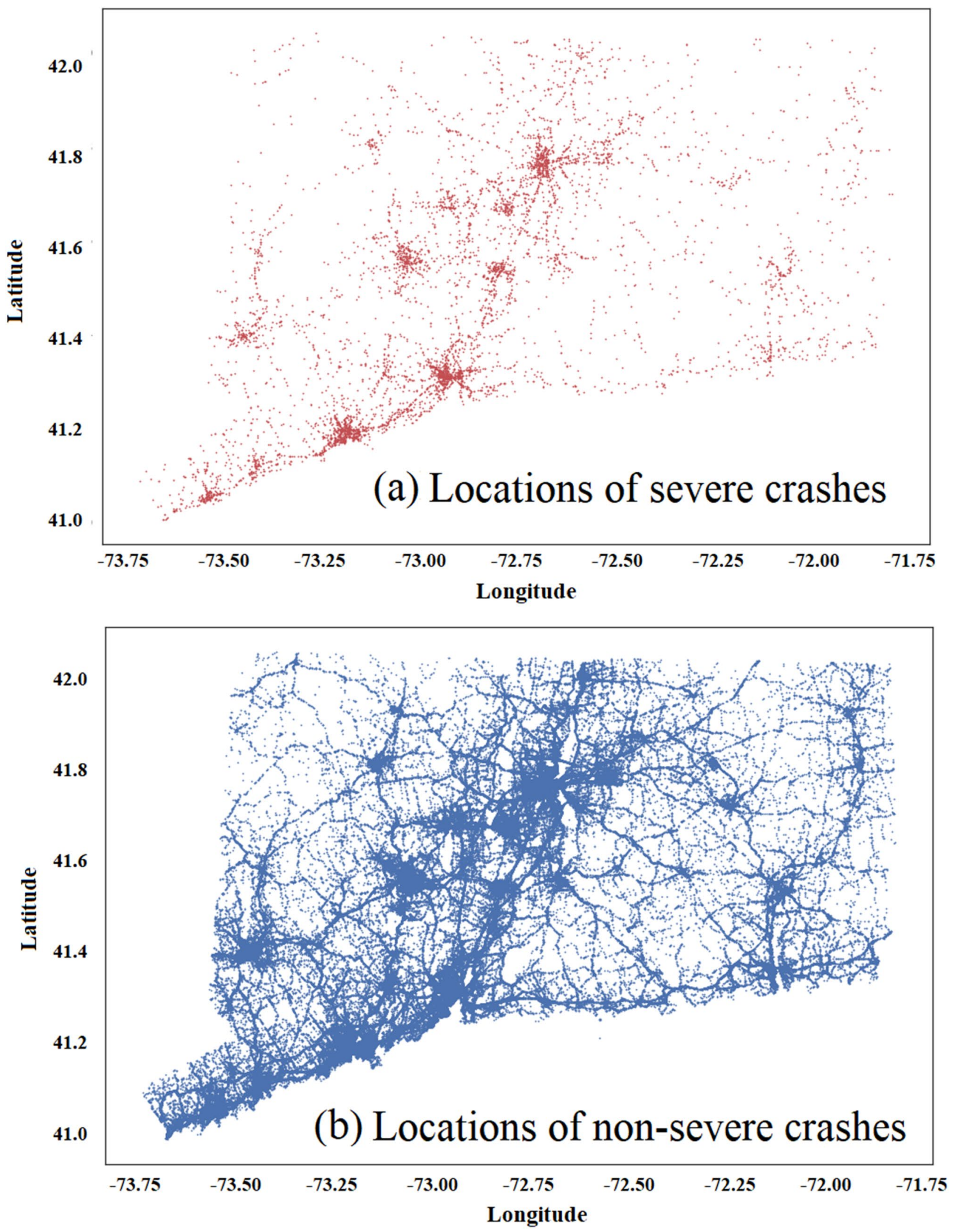

Fig. 1 Locations of the severe and non-severe crashes in study area (Connecticut) 
Fig. 2 Daily trend of severe and non-severe crashes for different years in Connecticut
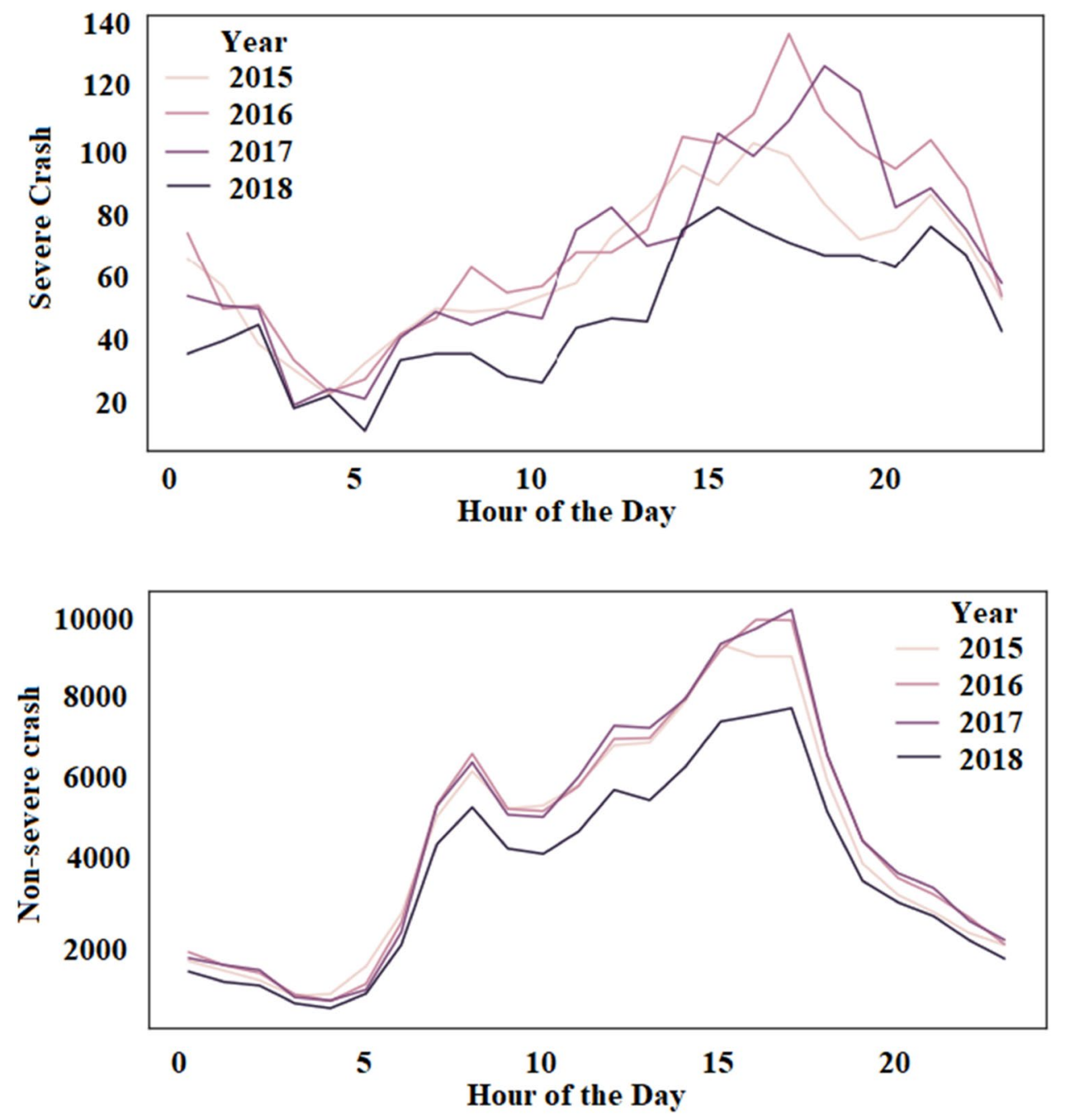

trees generated during the training process in case of regression or simply taking the mode of prediction in terms of classification.

\subsection{Bayesian additive regression trees (BART)}

Bayesian additive regression trees (BART) modeling approach involves estimating non-parametric functions using regression trees [12]. BART model is constructed as a summation of the $M$ number of regression trees that offer more flexibility compared to a single regression tree in fitting complex variable interactions and non-linearities [30]. Given that there is $M$ number of different regression trees, where a particular regression tree is denoted by $\mathrm{T}(\mathrm{X})$ with $X$ being the set of explanatory variables, the BART model can be formulated as:

$Y=T_{1}(X)+T_{2}(X)+T_{3}(X)+\ldots+T_{M}(X)+\epsilon$ (noise $)$. where $Y$ represents the crash severity as a response variable for the BART model. The BART model is based on the Bayesian modeling approach with regularization and likelihood estimation. Therefore, any individual tree cannot rule over the overall fit of the model. A detailed explanation of the BART model and its different components associated with this modeling approach can be found in Kapelner and Bleich [30]. BART can be configured for the classification problem with binary outcomes [16] and multiple classes as well [31]. In this analysis, the BART modeling technique is used for the same dataset as considered for RF models to compare the performance of these two ML techniques.

\subsection{Cross-validation experiment}

In this analysis, to develop the crash prediction models, we used K-fold cross-validation experiment (details in [8]) for assessing the effectiveness of the machine learning algorithms. After establishing the best features for the ML model, crash prediction probability was 
Fig. 3 A schematic representation of framework

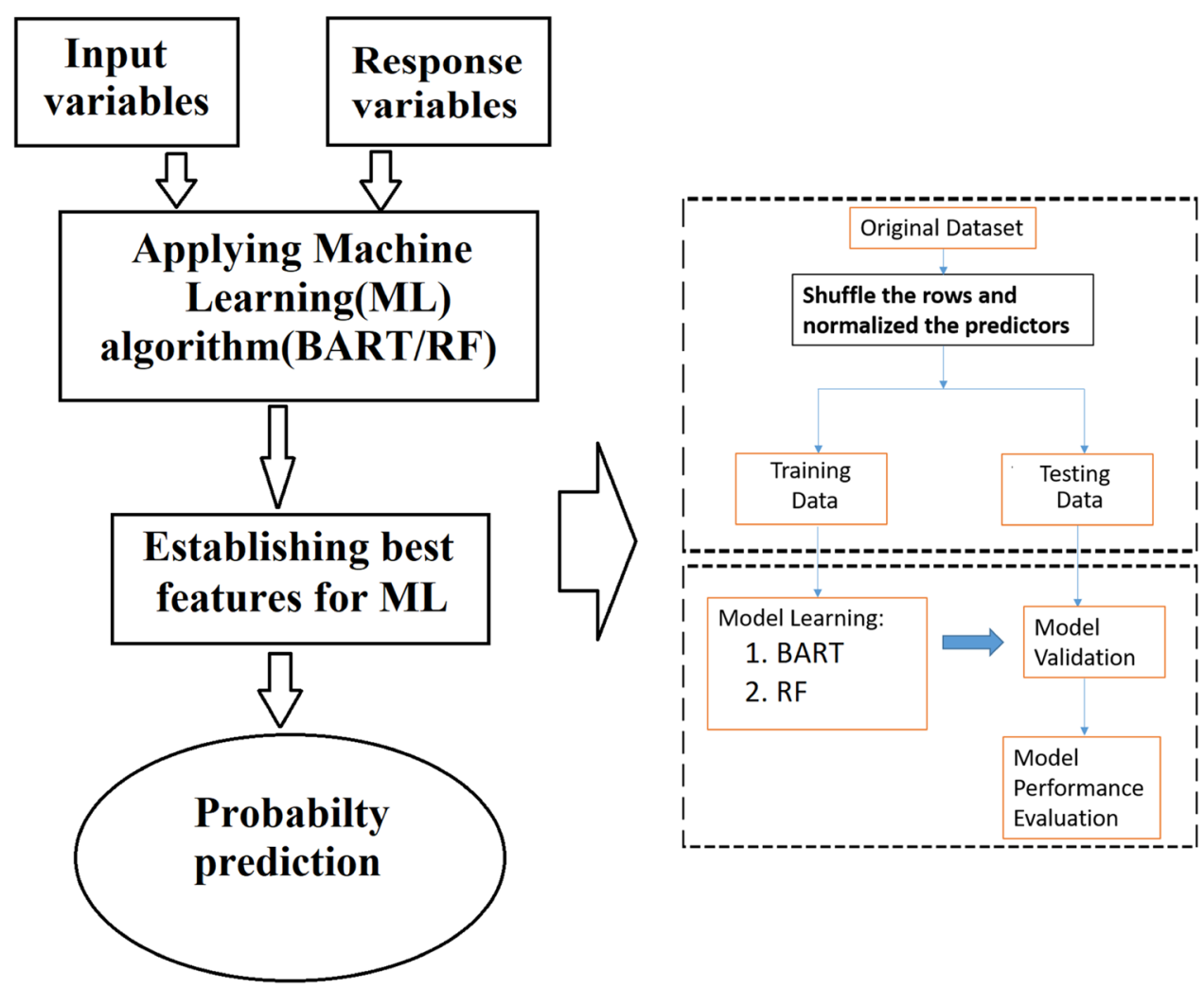

estimated. Initially, K-fold ( $\mathrm{K}=5$ ) cross-validation technique was used to develop the model in RF and BART applications. To create the crash prediction model, random holdout was applied five times over the study area. Specifically, for each holdout, dataset were split into $80 \%$ as training set and remaining $20 \%$ as validation set. We used "sample" function in R programming to shuffle the row indices of all the dataset to reorder the rows of the dataset randomly.

\subsection{Accuracy estimates for model prediction}

An accurate prediction of crash occurrence is represented by threat score, where the score of 1 specifies perfect prediction and calculated by the following equation:

Threat score $=\frac{\mathrm{TP}}{\mathrm{TP}+\mathrm{FN}}$

Note that true positive (TP) is the occurrence of crashes correctly identified, and false positive (FP) is the occurrence of crash identified by model but not true, and falsenegative (FN) is undetected crashes.

The mean square error (MSE) is a statistical metric used to measure the magnitude of the random error and is defined as:
$M S E=\frac{1}{n} \sum_{i=1}^{n}\left(\hat{y}_{i}-y_{i}\right)^{2}$

Here, $y_{i}$ is the observed crash, $\hat{y}_{i}$ is estimated crash for the model, and $\mathrm{n}$ is the total number of data points used in the calculation. MSE ranges from 0 to positive infinity with 0 indicating no random error. The importance of the predictor variables depends on the magnitude of the percentage increase in mean square error (\% IncMSE) of the model [7]. To estimate the \% IncMSE, we devised the following formula:

InCMSE $=\frac{\Delta_{b}-\Delta_{i}}{\Delta_{i}}$

where $\Delta_{b}$ indicates the MSE error metric of the model considering all predictors, and $\Delta_{i}$ represents the MSE error metric for the model excluding individual predictor.

\section{Results}

\subsection{Sensitivity analysis}

The predictor variables for RF technique were selected as "manner of crash", "weather condition", "route class", "hour of the day", "type of intersection", "light condition", "road 
surface condition", "month", "work zone related", "day of the work", and "school bus related". The selection was based on past research $[2,5,18,20,24,45]$ examining the factors related to crash severity prediction. Sensitivity analysis is conducted by calculating the magnitude of the percentage increase in mean square error (\%IncMSE) of the model [7] using Eq. (4), where higher values of \%IncMSE indicate higher importance of that predictor variable. Results from the variable importance test for the predictor variables are presented in Fig. 4. Specifically, manner of crash, and weather condition were ranked as the most important predictors with higher values of $\%$ IncMSE (65-75\%), showing their strong impact in model prediction. From a practical point of view, the severity of crashes, in terms of both bodily injury and property damage, depends mostly on the way the involved vehicles interact with each other or with the surrounding environment. For example, head-on collision between two vehicles moving in opposite directions increases the chance of fatality compared to side-swipe and other less impactful encounters. The weather condition is another exogenous variable associated with the roadway environment that brings in greater uncertainty as it impacts drivers' ability to safely maneuver the vehicles in the critical weather situation. Being a North England state, most weather-related crashes in Connecticut happen due to winter weather condition such as blizzards and heavy rain.

Route class, the hour of the day, intersection type, light, road surface condition, and month were also identified significant predictors with \% IncMSE 50-60\%, indicating the high importance in model prediction. Practically, route class plays an important role in determining crash severity, as the route class is defined based on the lane configuration, maximum allowable speed limit, type of access control, and surrounding land use. For example, chances of head-on collisions are higher in undivided highways compared to divided highways and having multiple lanes also increases the scope of interaction between vehicles leading to a higher possibility of crashes. Moreover, the hour of the day is the subsequent variable of importance, and the exploratory analysis of the crash dataset shows that severe accidents mostly happen during the afternoon peak hours (Fig. 2). The plausible reason behind this trend can be the fact that most drivers get exhausted in their work and this
Fig. 4 Variable importance plot, where \% IncMSE is the percentage increase in mean square error

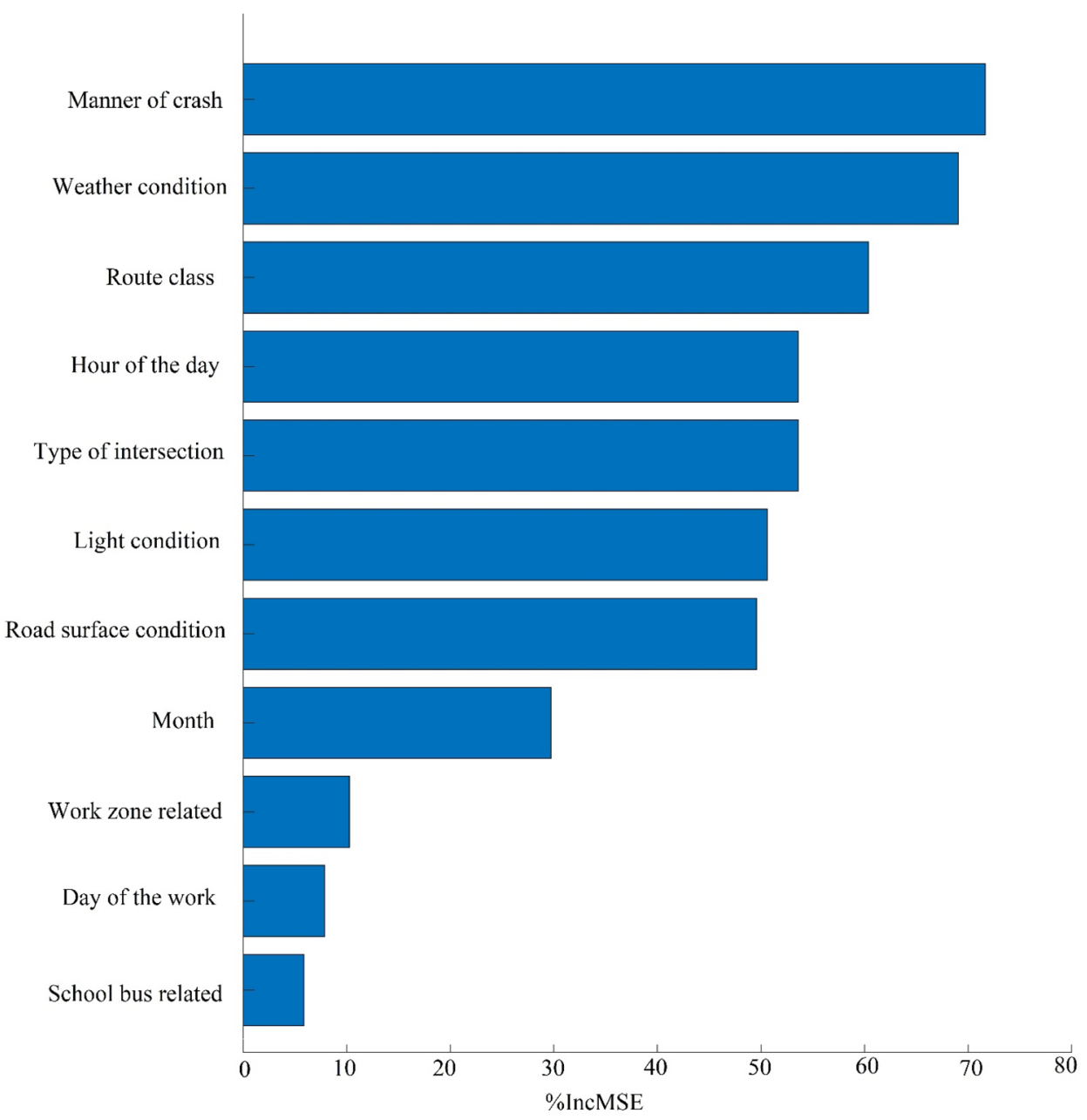

SN Applied Sciences 
tiredness results in reduced attention to driving and surrounding environment after work hours leading to more fatal crashes. Type of intersections is also a similar determinant of crash severity as there may arise confusion and misunderstanding among the drivers of involved vehicles due to increased points of conflict and poor judgments of drivers. Light and road surface conditions are equally important variables as these may impact the ability of drivers to control their vehicles and appropriately interact with the road environment.

Moreover, the other predictors (month, work zone, day of the work, and the presence of school buses) have lower but satisfactory \% IncMSE magnitude (5-30\%) which also confirms the relative importance of those variables in the model prediction. Most severe crashes happen during the winter months due to frequent adverse weather conditions and also in summer due to the high volume of traffic increasing the possibility of frequent interaction in the road environment. In the work zone and around the school bus, drivers usually become more cautious and tend to follow the traffic rules due to severe penalties associated with the offenses made by the drivers, resulting in a lower possibility of crashes. Lastly, the day of the work also shows marginal importance of crash severity prediction, since this variable does not directly affect the driving capability (e.g., people do not drive differently in different days of the week) and exogenous factors associated with the roadway environment. Finally, the study considered all the 11 predictor variables to predict the crash severity level.

\subsection{Evaluation results}

As it is illustrated in Fig. 5, the predicted probabilities obtained from the RF model show a promising trend with very high probabilities for a high number of events. Most events have prediction probabilities greater than $50 \%$ which indicates that the performance of RF models is reliable and satisfactory. In the case of BART, the trend is somehow not as good as the RF models. The prediction probabilities of almost all events are less than $50 \%$ which makes it a weaker competitor of the RF model as shown

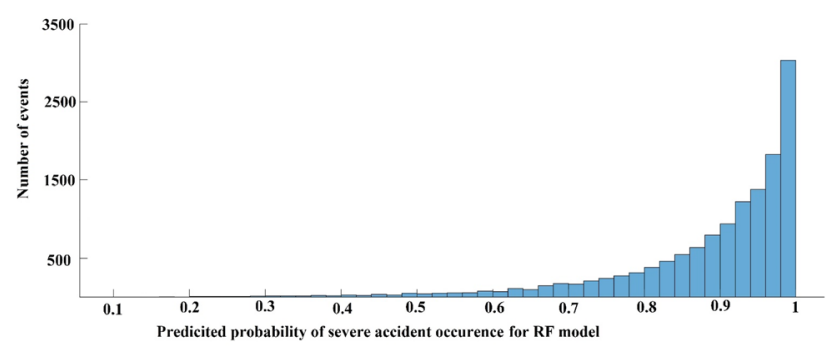

Fig. 5 Prediction probabilities using RF model in Fig. 6. Also, there are a lot of events where BART fails to predict the severity of crashes with a reasonable degree of probabilities. When the prediction probabilities obtained from RF and BART models are compared with each other, a perfect $y=x$ relationship should be observed as the models should calculate the same degree of prediction probabilities to be comparable with each other. However, as shown in Fig. 7, the slope of the regression between RF versus BART is found to be almost 1.7, which also indicates that RF model calculates higher prediction probabilities for most crashes compared to those of BART. The $R^{2}$ value is also very low indicating that the prediction probabilities of the BART model fit very loosely with those of RF models.

Figure 8 explains further how the RF model outperforms BART models in predicting crash severity with higher probabilities. The median of predicted probabilities of the RF

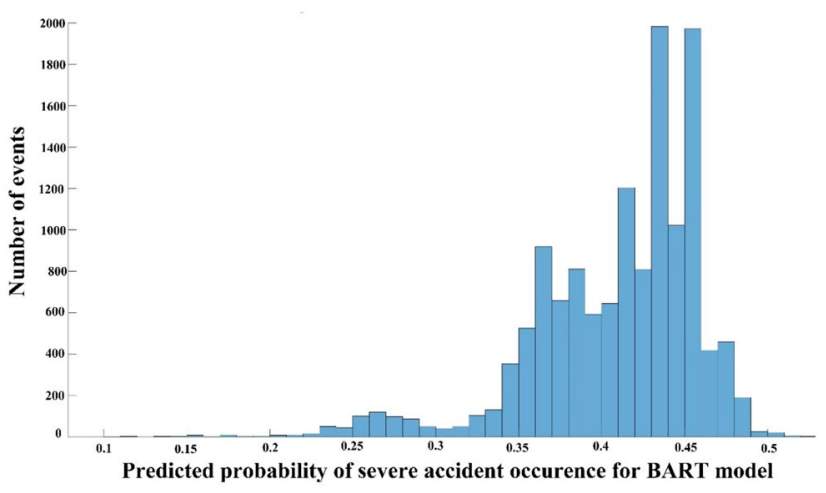

Fig. 6 Prediction probabilities using BART model

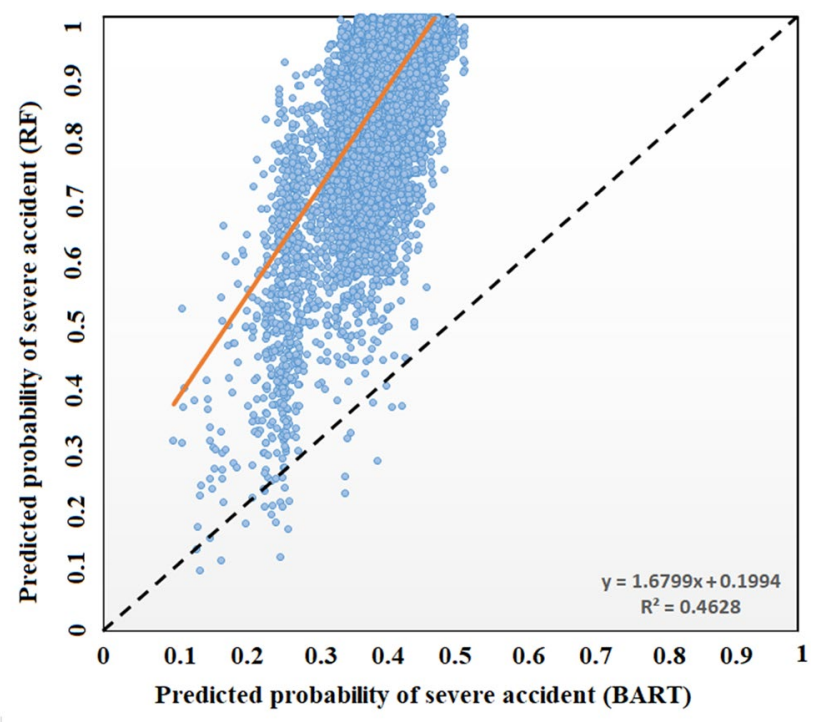

Fig. 7 Comparison of prediction probabilities obtained from RF and BART 


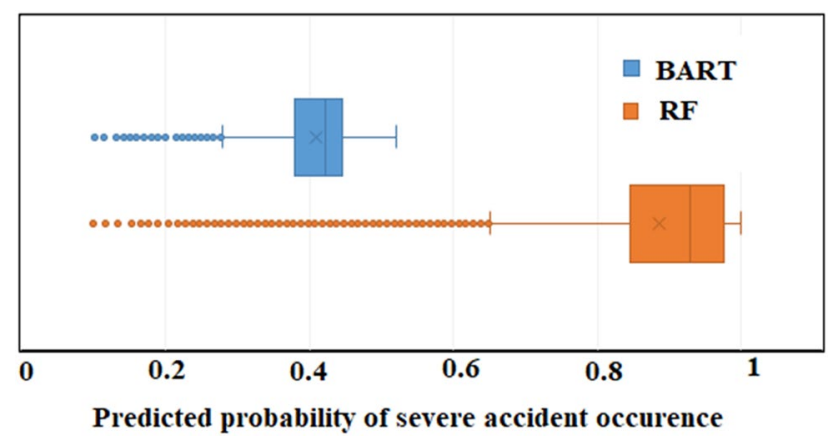

Fig. 8 Range of prediction probabilities obtained from RF and BART. In each box, the central mark is the median, and the edges are the first and third quartiles. The whiskers extended to the most extreme data points considered outlier using the orange dot symbol

Table 1 Threat score for training and testing for different techniques

\begin{tabular}{lll}
\hline Threat score & Training & Testing \\
\hline RF & 0.785 & 0.73 \\
BART & 0.673 & 0.61 \\
\hline
\end{tabular}

model is incredibly higher than that of BART models. One interesting observation in Fig. 8 is that the interquartile ranges do not overlap with each other being the case the 25 th percentile prediction probability of RF models is higher than the 75th percentile prediction probabilities of the BART model. Therefore, it can be concluded that $\mathrm{RF}$ model is more reliable to predict crash severity compared to BART model. Moreover, to demonstrate the performance of the individual models, the skill score is used in this study to examine how closely the model predicted crash that matches the observation data. BART and RF models estimate the probability of crash occurrence for both training and validation (Table 1). An accurate prediction is represented by the metric value close to 1. During the training of the RF model, the skill score was 0.785 which is higher than the BART produced skill score of 0.67 . Similarly, during the validation, we found a promising performance of RF models by exhibiting a higher skill score (0.73) than BART (0.61).

\section{Discussion and conclusions}

The predictability of crash from machine learning models exhibits considerable variation in pattern and magnitude, which results in significant differences in these two model simulations. Results presented in this paper demonstrated that the magnitude and dynamics of uncertainty in crash severity depend not only on the weather condition but also on the other crash associated auxiliary variables. Knowledge-based crash severity prediction has always been the principal focus of safety professionals and emergency responders for appropriate policy making and resource management. Over the years, different statistical methodologies have been explored in various research efforts to find out efficient crash severity prediction models. As technology is advancing and computing has started becoming more efficient, machine learning-based models for crash severity are being brought into the light for more accurate prediction models. However, some machine learning methodologies provide increased efficiency and better performance compared to others.

To explore different machine learning methodologies for crash severity prediction, this study considered two machine learning applications-RF and BART for performance comparison. The study found that RF model considering weather conditions have higher prediction probabilities for determining crash severity compare to BART model predictions. Various analyses of prediction probabilities provided useful insights into the models' capability to generate predictions that are consistent with observed data. A variable importance approach confirms that manner of crash and weather conditions are two major variables that can feed useful information in the modeling estimation process. The study finds that stakeholders can use the RF model with confidence to obtain a better prediction of crash severity that enable them to facilitate appropriate emergency responses and support essential preventive measures. Further exploration can be useful to compare more machine learning modeling approaches that might have better performance and efficiency. It might also be reasonable to compare their results with statistical approaches to compile a comprehensive comparison, so the stakeholder can make knowledgeable decisions for their operational strategies.

Acknowledgements We gratefully acknowledge the Connecticut Crash Data Repository (CTCDR) as the primary source of crash data used in this study.

\section{Compliance with ethical standards}

Conflict of interest The authors of this study declare that they have no conflict of interest in the core methodology developed for this study, interpretation of results, and presentation of the findings.

\section{References}

1. Abellán J, López G, de Oña J (2013) Analysis of traffic accident severity using decision rules via decision trees. Expert Syst Appl 40:6047-6054

2. Anderson TK (2009) Kernel density estimation and K-means clustering to profile road accident hotspots. Accid Anal Prev 41:359-364 
3. Abdel-Aty MA, Abdelwahab HT (2004) Predicting injury severity levels in traffic crashes: a modeling comparison. J Transp Eng 130:204-210

4. Abdelwahab HT, Abdel-Aty MA (2001) Development of artificial neural network models to predict driver injury severity in traffic accidents at signalized intersections. Transp Res Rec 1746:6-13

5. Abdul-Aziz HM, Ukkusuri SV, Hasan S (2010) Exploring the determinants of pedestrian-vehicle crash severity in New York City. Accid Anal Prev 50:1298-1309

6. Amit $Y$, Geman D (1997) Shape quantization and recognition with randomized trees. Neural Comput 7:1545-1588

7. Breiman $L$ (2001) Random forests. Mach Learn 45:5-32

8. Bhuiyan MAE, Begum F, Ilham SJ, Khan RS (2019) Advanced wind speed prediction using convective weather variables through machine learning application. Appl Comput Geosci 1:10002

9. Bhuiyan MAE, Nikolopoulos El, Anagnostou EN (2019) Machine learning-based blending of satellite and reanalysis precipitation datasets: a multi-regional tropical complex terrain evaluation. J Hydrometeorol. https://doi.org/10.1175/JHM-D-19-0073.1

10. Bhuiyan MAE, Nikolopoulos El, Anagnostou EN, QuintanaSeguí P, Barella-Ortiz A (2018) A nonparametric statistical technique for combining global precipitation datasets: development and hydrological evaluation over the Iberian Peninsula. Hydrol Earth Syst Sci 22:1371-1389. https://doi.org/10.5194/ hess-22-1371-2018

11. Bhuiyan MAE, Anagnostou EN, Kirstetter PE (2017) A nonparametric statistical technique for modeling overland TMI (2A12) rainfall retrieval error. IEEE Geosci Remote $S$ 14:1898-1902

12. Chipman HA, George El, McCulloch RE (2010) Bart: Bayesian additive regression trees. Ann Appl Stat 4:266-298

13. Chang L-Y, Wang H-W (2006) Analysis of traffic injury severity: an application of non-parametric classification tree techniques. Accid Anal Prev 38:1019-1027

14. Chong M, Abraham A, Paprzycki M (2005) Traffic accident analysis using machine learning paradigms. Informatica 29:89-98

15. Cerrai D, Wanik DW, Bhuiyan MAE, Zhang X, Yang J, Frediani MEB, Anagnostou EN (2019) Predicting storm outages through new representations of weather and vegetation. IEEE Access 7:29639-29654

16. Chipman HA, George El, McCulloch RE (2012) Bayesian additive regression trees. Ann Appl Stat. https://doi.org/10.1214/09AOAS285

17. Delen D, Sharda R, Bessonov M (2006) Identifying significant predictors of injury severity in traffic accidents using a series of artificial neural networks. Accid Anal Prev 38:434-444. https:// doi.org/10.1016/J.AAP.2005.06.024

18. Davis $G$ (2010) Relating severity of pedestrian injury to impact speed in vehicle-pedestrian crashes: simple threshold model. Transp Res Rec 1773:108-113

19. De Oña J, López G, Mujalli R, Calvo FJ (2013) Analysis of traffic accidents on rural highways using latent class clustering and Bayesian networks. Accid Anal Prev 51:1-10. https://doi. org/10.1016/J.AAP.2012.10.016

20. Das A, Abdel-Aty M, Pande A (2009) Using conditional inference forests to identify the factors affecting crash severity on arterial corridors. J Saf Res 40:317-327. https://doi.org/10.1016/J. JSR.2009.05.003

21. Eluru N, Bagheri M, Miranda-Moreno LF, Fu L (2012) A latent class modeling approach for identifying vehicle driver injury severity factors at highway-railway crossings. Accid Anal Prev 47:119-127. https://doi.org/10.1016/J.AAP.2012.01.027

22. EIDessouki WM, Ivan JN, Anagnostou EN, Sadek AW, Zhang $C$ (2004) Using relative risk analysis to improve connecticut freeway traffic safety under adverse weather conditions, New England (Region One) UTC. US Department of Transportation, Publication\#: UCNR, 14-5
23. Goodwin LC, Pisano P (2003) Weather-related crashes on U.S. highways in 2001, Mitretek systems, prepared for the FHWA Road Weather Management Program

24. Harb R, Yan X, Radwan E, Su X (2009) Exploring precrash maneuvers using classification trees and random forests. Accid Anal Prev 41:98-107. https://doi.org/10.1016/J.AAP.2008.09.00

25. Ho TK (1995) Random decision forests

26. Ho TK (1998) The random subspace method for constructing decision forests. IEEE Trans Pattern Anal Mach Intell. https://doi. org/10.1109/34.709601

27. He J, Wanik D, Hartman B, Anagnostou E, Astitha M, Frediani MEB (2017) Nonparametric tree-based predictive modeling of storm damage to power distribution network. Risk Anal 37:441-458. https://doi.org/10.1111/risa.12652

28. Hastie T, Tibshirani R, Friedman J (2005) The elements of statistical learning: data mining, inference and prediction. Math Intell 27:83-85

29. Kashani AT, Mohaymany AS (2011) Analysis of the traffic injury severity on two-lane, two-way rural roads based on classification tree models. Saf Sci 49:1314-1320. https://doi.org/10.1016/J. SSCI.2011.04.019

30. Kapelner A, Bleich J (2016) BartMachine: machine learning with Bayesian additive regression trees. J Stat Softw. https:// doi.org/10.18637/jss.v070.i04

31. Kindo BP, Wang H, Peña EA (2016) Multinomial probit bayesian additive regression trees. Stat. https://doi.org/10.1002/sta4.110

32. Kaplan S, Prato CG (2013) Cyclist-motorist crash patterns in denmark: a latent class clustering approach. Traffic Injury Prev 14:725-733. https://doi.org/10.1080/15389588.2012.759654

33. Li Z, Liu P, Wang W, Xu C (2012) Using support vector machine models for crash injury severity analysis. Accid Anal Prev 45:478486. https://doi.org/10.1016/J.AAP.2011.08.016

34. Mohamed MG, Saunier N, Miranda-Moreno LF, Ukkusuri SV (2013) A clustering regression approach: a comprehensive injury severity analysis of pedestrian-vehicle crashes in New York, US and Montreal, Canada. Saf Sci 54:27-37. https://doi. org/10.1016/J.SSCI.2012.11.001

35. Montella A, Aria M, D'Ambrosio A, Mauriello F (2012) Analysis of powered two-wheeler crashes in Italy by classification trees and rules discovery. Accid Anal Prev 49:58-72. https://doi. org/10.1016/J.AAP.2011.04.025

36. Mujalli RO, de Oña J (2011) A method for simplifying the analysis of traffic accidents injury severity on two-lane highways using Bayesian networks. J Saf Res 42:317-326. https://doi. org/10.1016/J.JSR.2011.06.010

37. Mauro R, De Luca M, Dell'Acqua G (2013) Using a k-means clustering algorithm to examine patterns of vehicle crashes in before-after analysis. Modern Appl Sci 7:11

38. Malyshkina NV, Mannering FL (2010) Empirical assessment of the impact of highway design exceptions on the frequency and severity of vehicle accidents. Accid Anal Prev 42:131-139. https ://doi.org/10.1016/J.AAP.2009.07.013

39. Pisano PA, Goodwin LC, Rossetti MA (2008) US highway crashes in adverse road weather conditions. In: 24th conference on international interactive information and processing systems for meteorology, oceanography and hydrology, New Orleans, LA

40. Savolainen PT, Mannering FL, Lord D, Quddus MA (2011) The statistical analysis of highway crash-injury severities: a review and assessment of methodological alternatives. Accid Anal Prev 43:1666-1676. https://doi.org/10.1016/J.AAP.2011.03.025

41. Shankar V, Mannering F, Barfield W (1996) Statistical analysis of accident severity on rural freeways. Accid Anal Prev 28:391-401. https://doi.org/10.1016/0001-4575(96)00009-7

42. Ye F, Lord D (2014) Comparing three commonly used crash severity models on sample size requirements: multinomial logit, 
ordered probit and mixed logit models. Anal Methods Accid Res 1:72-85. https://doi.org/10.1016/J.AMAR.2013.03.001

43. Yasmin S, Eluru N (2013) Evaluating alternate discrete outcome frameworks for modeling crash injury severity. Accid Anal Prev 59:506-521. https://doi.org/10.1016/J.AAP.2013.06.040

44. Yang F, Wanik DW, Cerrai D, Bhuiyan MAE, Anagnostou EN (2020) Quantifying uncertainty in machine learning-based power outage prediction model training. MDPI, sustainability

45. Zhang C, Ivan JN, Jonsson T (2007) Collision type categorization based on crash causality and severity analysis. In: 86th annual meeting of the transportation research board 2007. Transportation Research Board, Washington DC, USA

Publisher's Note Springer Nature remains neutral with regard to jurisdictional claims in published maps and institutional affiliations. 